\title{
RELATIVELY UNIFORM BANACH LATTICES ${ }^{1}$
}

\section{ANDREW WIRTH}

ABSTRACT. Sequential relative uniform and norm convergence agree in a Banach lattice, if and only if it is equivalent to an $M$ space.

Let $(E, \leq,\|\cdot\|)$ be a Banach lattice. The sequence $\left\{f_{n}\right\}$ is said to be relatively uniformly (abbreviated $\mathrm{ru}$ ) convergent to $f$, if there exists $u>0, \lambda_{n} \epsilon$ $\mathbf{R}, \lambda_{n} \downarrow 0$, such that $\left|f_{n}-f\right| \leq \lambda_{n} u$ for all $n$. If there exist an increasing sequence $\left\{g_{n}\right\}$ and a decreasing sequence $\left\{h_{n}\right\}$ such that $g_{n} \leq f_{n} \leq h_{n}$ for all $n$, and $\vee g_{n}=\bigwedge h_{n}=f$, we say $\left\{f_{n}\right\}$ is order (abbreviated o) convergent to $f$. We say two Banach lattices are equivalent if there exists a vector lattice isomorphism under which the norms are equivalent. Call E relatively uniform if norm and ru convergence agree, for sequences, on $E$.

Firstly we indicate why the corresponding notion for nets is rather trivial. We say the net $\left\{f_{a}\right\}_{a \in A}$ ru converges to $f$ if there exists $u>0$, such that given any $\epsilon>0$ there exists $\alpha_{0} \epsilon A$ with $\left|f_{a}-f\right| \leq \epsilon u$ for all $\alpha \geq \alpha_{0}$.

Proposition 1. $E$ is net relatively uniform if and only if $E$ contains a strong unit.

Proof. Suppose $E$ is net relatively uniform. If $\alpha, \beta \in E \backslash\{0\}$ write $\alpha \preccurlyeq \beta$ to mean $\|\alpha\| \geq\|\beta\|$. Let $f_{\alpha}=\alpha$, then $\|\cdot\|-\lim f_{a}=0$, so ru-lim $f_{\alpha}=$ 0 . Hence $E$ contains a strong unit.

Conversely suppose $E$ contains a strong unit $e$. The norms $\|\cdot\|$ and $\|\cdot\|_{e}$ are equivalent, by Birkhoff $\left[1\right.$, Lemma 5, p. 367], where $\|f\|_{e}=\inf \{\lambda$ : $|f| \leq \lambda e\}$.

From now on we only consider sequential convergence.

The following result is contained, implicitly, in Leader [3].

Lemma 1 (Leader). If $E$ is relatively uniform then it is equivalent to an M space.

Proof. By [3, Theorem 4] it follows that $\|\cdot\|$ is equivalent to an $M$ norm if and only if, for every sequence $\{p(n)\}$ of positive integers, and for every sequence $\left\{f_{n}\right\}$ such that $\left\|f_{n}\right\| \cdot \rightarrow 0$, we have

$$
\left\|\left|f_{n}\right| \vee\left|f_{n+1}\right| \vee \cdots \vee\left|f_{n+p(n)}\right|\right\| \rightarrow 0
$$

Received by the editors June 19, 1974.

AMS (MOS) subject classifications (1970). Primary 46A40.

Key words and phrases. Banach lattice, relative uniform convergence, order convergence, $M$ space, $L^{P}$ space.

1 This research was supported by a Junior Rothmans Fellowship. 
If $E$ is relatively uniform and $\left\|f_{n}\right\| \rightarrow 0$, then $\left|f_{n}\right| \vee\left|f_{n+1}\right| \vee \cdots \vee$ $\left|f_{n+p(n)}\right| \leq \lambda_{n} u$, hence (1) holds.

Lemma 2. In an M space a norm convergent sequence has a supremum.

Proof. Let $\left\{f_{n}\right\}$ be a norm convergent sequence. Denote $f_{1} \vee \cdots \vee f_{n}$ by $g_{n}$. We show $\left\{g_{n}\right\}$ is Cauchy. Let $n>m$, then

$$
\begin{aligned}
g_{n}-g_{m} & =g_{m} \vee f_{m+1} \vee \cdots \vee f_{n}-g_{m} \\
& =\left(f_{m+1}-g_{m}\right)^{+} \vee \cdots \vee\left(f_{n}-g_{m}\right)^{+} .
\end{aligned}
$$

Now

$$
f_{i}-g_{m}=f_{i}-f_{1} \vee \cdots \vee f_{m}=\left(f_{i}-f_{1}\right) \wedge \cdots \wedge\left(f_{i}-f_{m}\right) \text {, }
$$

so $\left(f_{i}-g_{m}\right)^{+} \leq\left(f_{i}-f_{m}\right)^{+}$. Hence

$$
\begin{aligned}
\left\|g_{n}-g_{m}\right\| & =\left\|\bigvee_{i=m+1}^{n}\left(f_{i}-g_{m}\right)^{+}\right\| \leq \bigvee_{i=m+1}^{n}\left(f_{i}-f_{m}\right)^{+} \| \\
& =\bigvee_{i=m+1}^{n}\left\|\left(f_{i}-f_{m}\right)^{+}\right\| \leq \bigvee_{i=m+1} \bigvee_{i}^{n}\left\|f_{i}-f_{m}\right\| .
\end{aligned}
$$

Thus $\left\{g_{n}\right\}$ is Cauchy. Let $\|\cdot\|-\lim g_{n}=g$; it then follows, from the fact that the positive cone is (norm) closed, that $g=\bigvee_{n=1}^{\infty} f_{n}$.

Proposition 2。 The following statements are pairwise equivalent:

(i) $E$ is relatively uniform;

(ii) $E$ is equivalent to an M space;

(iii) $\left\{f_{n}\right\}$ norm converges implies that $\bigvee f_{n}$ exists;

(iv) $\left\{f_{n}\right\}$ norm converges implies that $\wedge f_{n}$ exists;

(v) $\left\{f_{n}\right\}$ norm converges implies that $\left\{f_{n}\right\}$ order converges.

Proof. (i) implies (ii) is Lemma 1, (ii) implies (iii) is Lemma 2.

(iii) implies (v). If $\left\|f_{n}\right\| \rightarrow 0$ then $u=V_{\left\|f_{n}\right\|>0}\left\|f_{n}\right\|^{-1 / 2}\left|f_{n}\right|$ exists. So $f_{n}$ ru converges to 0 . Since $E$ is archimedean, (v) follows.

(iii) and (iv) are equivalent. If $\left\{f_{n}\right\}$ norm converges then so does $\left\{-f_{n}\right\}$, and $\vee-f_{n}=-\wedge f_{n}$.

(v) implies (i). Suppose $\left\|f_{n}\right\| \rightarrow 0$ then $\left\|f_{n}\right\|^{-1 / 2}\left|f_{n}\right|$ norm converges to 0 , so by (v) there exists $v \geq\left\|f_{n}\right\|^{-1 / 2}\left|f_{n}\right|$, hence (i) holds (we may assume without loss of generality that $\left\|f_{n}\right\|>0$ for all $n$ ).

A Banach lattice is called an $L^{p}$ space if $|f| \wedge|g|=0$ implies that $\|f+g\|=\left(\|f\|^{p}+\|g\|^{p}\right)^{1 / p}$ where $1 \leq p<\infty$ (Birkhoff [1, p. 378]).

Corollary 1. An infinite dimensional $L^{p}$ space is not relatively uniform, and is not equivalent to an $M$ space. 
Proof. Suppose there exists an infinite dimensional relatively uniform $L^{p}$ space, E. By Zaanen [4, Theorem 4.3] there exists a sequence $\left\{f_{n}\right\}$ of strictly positive pairwise orthogonal elements in $E$. We may assume that $\left\|f_{n}\right\|=n^{-1 / p}$. So ru-lim $f_{n}=0$, and hence there exists $u \in E$ such that $f_{n} \leq u$ for all $n$. So

$$
\begin{aligned}
\|u\| & \geq\left\|f_{1} \vee \cdots \vee f_{n}\right\|=\left\|f_{1}+\cdots+f_{n}\right\| \\
& =\left(\left\|f_{1}\right\|^{p}+\cdots+\left\|f_{n}\right\|^{p}\right)^{1 / p}=\left(\sum_{k=1}^{n} \frac{1}{k}\right)^{1 / p} \rightarrow \infty,
\end{aligned}
$$

giving the required contradiction. The rest follows from Proposition 2.

Corollary 2. In an infinite dimensional $L^{p}$ space every l-ideal, generated by countably many elements, is proper.

Proof. Suppose $E$ is an infinite dimensional $L^{p}$ space, containing a sequence $\left\{f_{n}>0\right\}$ which is contained in no proper $l$-ideal. Let $e=$ $\sum_{n=1}^{\infty} n^{-2}\left\|f_{n}\right\|^{-1} f_{n}$, then $e$ is a strong unit. With the equivalent norm $\|\cdot\|_{e}$ (see Proposition 1) $E$ becomes an $M$ space, with unity $e$. The rest follows by Corollary 1.

In the case of $l^{1}$ the above reduces to the well-known result, that even with countably many 'test' series the comparison test is not effective on all positive term series; see Knopp [2].

\section{REFERENCES}

1. L. G. Birkhoff, Lattice the ory, 3rd ed., Amer, Math. Soc. Colloq. Publ., Vol. 25, Amer. Math. Soc., Providence, R. I., 1967. MR 37 \#2638.

2. K. Knopp, Theory and application of infinite series, 2nd English ed., Blackie, London, 1951.

3. S. Leader, Sequential convergence in lattice groups, Problems in Analysis, (R. C. Gunning, editor), Princeton, 1970, pp. 273-290.

4. A. C. Zaanen, Stability of order convergence and regularity in Riesz spaces, Studia Math. 31 (1968), 159-172. MR 39 \#1944.

DEPARTMENT OF MATHEMATICS, MONASH UNIVERSITY, CLAYTON 3168, VICTORIA, AUSTRALIA 